\title{
Endogenous glucocorticoids promote the expansion of myeloid-derived suppressor cells in a murine model of trauma
}

\author{
KUN ZHANG $^{1}{ }^{1}$, XIANGJUN BAI ${ }^{1}$, RENJIE LI ${ }^{1}$, ZHENGZHENG XIAO $^{2}$, \\ JIAJUN CHEN $^{1}$, FAN YANG ${ }^{1}$ and ZHANFEI LI ${ }^{1}$ \\ Departments of ${ }^{1}$ Trauma Surgery and ${ }^{2}$ Neurosurgery, Tongji Hospital, Tongji Medical \\ College, Huazhong University of Science and Technology, Wuhan, P.R. China
}

Received March 9, 2012; Accepted May 11, 2012

DOI: 10.3892/ijmm.2012.1014

\begin{abstract}
Stress-dose of glucocorticoid has been demonstrated to be beneficial for trauma patients in clinical studies. Recently, a heterogeneous population of myeloid cells with immunosuppressive activity named myeloid-derived suppressor cells (MDSCs) has been found to accumulate in the trauma host and can be induced by glucocorticoids in vitro. In order to explore the effect of endogenous glucocorticoids on MDSCs under trauma conditions, we blocked the glucocorticoid signal in a murine trauma model using the antagonist of the glucocorticoid receptor RU486 (mifepristone). We found for the first time that RU486 not only blunted MDSC expansion induced by trauma in the spleen, peripheral blood and bone marrow especially at $6 \mathrm{~h}$ after traumatic stress but also decreased the survival rate from 100 to $20 \%$ in traumatic mice within 7 days. Moreover, neither MDSCs producing arginase-1 nor the morphological characterization of trauma-induced MDSCs was affected by the blockage of the glucocorticoid receptor. Our results suggest that endogenous glucocorticoids may promote MDSCs expansion in a murine trauma model and MDSCs may be beneficial for the trauma host.
\end{abstract}

\section{Introduction}

Trauma patients are often diagnosed as having systemic inflammatory response syndrome (SIRS), which usually leads to nonreversible multi-organ dysfunction syndrome (MODS) and eventually to patient death. Thus, controlling the SIRS after trauma is of high value in the care of trauma patients (1-5). Glucocorticoids, which have been widely used in treating inflammatory disorders, are well known for their regulation of the immune system. Due to their suppressing effects on excessive immune response and SIRS after trauma, glucocorticoids

Correspondence to: Dr Zhanfei Li, Department of Trauma Surgery, Tongji Hospital, Tongji Medical College, Huazhong University of Science and Technology, Jie Fang Avenue 1095, Wuhan 430030, P.R. China

E-mail: zhanfeilee@yahoo.cn

Key words: glucocorticoid, myeloid derived suppressor cells, trauma are supposed to be theoretically beneficial in treating trauma patients $(6,7)$. Moreover, a clinical study has demonstrated that a stress-does of hydrocortisone improves outcome in trauma patients, especially in patients with trauma-related corticosteroid insufficiency $(6,8)$. However, the antiinflammatory action of glucocorticoids is very complicated (9). In this study, we mainly focused on the relation between glucocorticoid and the immunoregulatory myeloid derived suppressor cells (MDSCs) under traumatic conditions.

MDSCs are a heterogeneous population of cells that consists of mature or immature myeloid cells, which express $\mathrm{CD}_{11} \mathrm{~b}^{+} / \mathrm{Gr}-1^{+}$markers and exert immune regulatory function in cancer, autoimmune diseases and chronic or acute inflammation (10-14). It has been reported that MDSCs expansion can be induced by physical injury in the spleen of a trauma model $(15,16)$. Moreover, trauma-induced MDSCs (TIMDSCs) are considered to take responsibility for immune suppression and high susceptibility after trauma via producing arginase-1 which mediates arginine depletion and results in T-cells dysfunction $(15,17)$. However, the precise role of MDSCs in trauma remains unclear. Some studies advocate that MDSCs expansion may play a positive role and be a part of the selfprotection in trauma (18).

Recent studies have reported that glucocorticoids can induce a monocyte subsite, which has immune suppressive function and resembles MDSCs in vitro (19-21). However, the effects of endogenous glucocorticoid on TIMDSCs in vivo are still unknown. Therefore, we hypothesized that endogenous glucocorticoids might be involved in TIMDSCs expansion, which may be a mechanism underlying the protective role of endogenous glucocorticoids under traumatic condition. In this study, we found that a glucocorticoid receptor blocker RU486 inhibited TIMDSCs expansion in the spleen, peripheral blood and bone marrow without affecting their characterization. Our study provides the first evidence that endogenous glucocorticoids are invovled in TIMDSCs expansion in a murine trauma model and suggests that TIMDSCs may correlate with the protective role of endogenous glucocorticoids in trauma host.

\section{Materials and methods}

Mice. Male BALB/c mice, 6 to 8 week of age, were obtained from the Center of Medical Experimental Animals of Hubei 
Province (Wuhan, China) and the study was approved by the Animal Care and Use Committee of Tongji Medical College. Mice were housed four per cage and were fed with food and water ad libitum under a 12 -h light/dark cycle at $20-22^{\circ} \mathrm{C}$ in a pathogen-free facility. Mice underwent an acclimation period of 2 weeks before experiment.

Mouse traumatic stress model. Mice were divided into three groups randomly: Group 1 received anesthesia only, Group 2 underwent traumatic stress, Group 3 received RU486 (SigmaAldrich) $(30 \mathrm{mg} / \mathrm{kg}$; i.p.) $30 \mathrm{~min}$ before traumatic stress. The traumatic stress was mimicked using an abdominal surgery reported by Makarenkova et al (15). Animals were sacrificed at 6,12 and $24 \mathrm{~h}$ after surgery, and spleen, bone marrow and peripheral blood were taken for cell harvest.

Isolation of $\mathrm{Gr}-\mathrm{I}^{+}$cells. The spleen, bone marrow and peripheral blood derived from the mice of the three groups were made into a single-cell suspension, respectively. Erythrocytes were depleted by RBC lysing buffer (Sigma-Aldrich), and all the cells were washed and resuspended in MACS buffer (1X PBS supplemented with $2 \mathrm{mM}$ EDTA and $0.5 \% \mathrm{BSA}$ ). $\mathrm{Gr}-1^{+}$cells were sorted using corresponding MACS magnetic microbeads (Miltenyi Biotec). The purity of $\mathrm{Gr}-1^{+}$cells ranged between 85 and $95 \%$.

Flow cytometry analysis. Harvested cells were suspended in FACS medium (1X PBS supplemented with $0.1 \%$ BSA and $0.1 \% \mathrm{NaN}_{3}$ ) and stained with FITC-labeled anti-mouse CD11b, and PE-labeled GR1 (eBioscience) according to the standard procedure. All staining procedures were performed on ice. The stained cells were counted using a FACScan flow cytometer (BD Biosciences).

Western blot analysis. Total protein from Gr-1 cells was separated on 10-12\% SDS-PAGE gels and electro blotted onto nitrocellulose membranes. The protein of the mouse liver served as a positive control. The nitrocellulose membrane was blocked in TBST (Tris-buffered saline with $0.5 \%$ Tween-20) containing $5 \%$ non-fat milk, and incubated with rabbit $\mathrm{IgG}$ anti-mouse arginase-1 primary antibodies (Santa Cruz Biotechnology, Inc., Santa Cruz, CA) overnight at $4^{\circ} \mathrm{C}$. The membrane was washed and incubated with a secondary antibody for $1 \mathrm{~h}$. Then the membrane was stripped, washed and incubated with goat anti-mouse $\beta$-actin Ab (Santa Cruz Biotechnology, Inc.), as an internal control for total protein concentration. After being washed, the membrane was incubated with secondary antibody for another $1 \mathrm{~h}$. ECL substrate (Pierce) was used for signal detection of both arginase- 1 and $\beta$-actin.

Morphological analysis of MDSCs. To observe the morphology of MDSCs, $10^{5}$ enriched Gr- $1^{+}$splenocytes were stained by One Step II Wright-Giemsa Stain Solution (Criterion Sciences). The $\mathrm{Gr}-1^{+}$splenocytes suspension was dropped onto a slide air-dried at room temperature.

Immunohistochemical staining. For immunohistochemistry, paraffin sections of spleens were deparaffinized and rehydrated by successive washes with xylene and graded ethanol. To block endogenous peroxidase, $3 \%$ hydrogen peroxide was

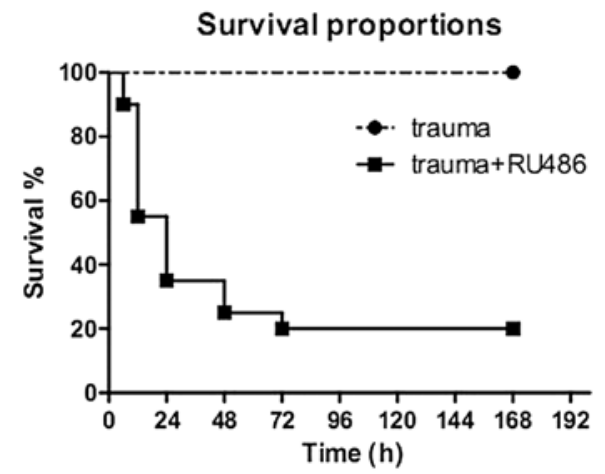

Figure 1. Survival proportions after trauma ( $n=20$ per group).

used. Antigen retrieval was performed in a microwave oven in $100 \mathrm{mM}$ sodium citrate buffer ( $\mathrm{pH} \mathrm{6.0)}$ ) for $10 \mathrm{~min}$ and then cooled to room temperature. Subsequently, slides were incubated with $10 \%$ normal serum followed by the primary anti-Gr-1 antibody (eBioscience) incubated overnight at $4^{\circ} \mathrm{C}$. Then the slides were incubated with biotinylated secondary antibody for $30 \mathrm{~min}$, and streptavidin-conjugated horseradish peroxidase (Santa Cruz Biotechnology, Inc.) was used for immunohistochemical staining. Digital images were acquired using an Olympus Microscope.

Statistical analysis. The significance of differences between the experimental groups was evaluated using one-way ANOVA (SigmaStat software; Jandel Scientific). Student's t-test was used for a single comparison of the two groups. P-value $<0.05$ was considered significant. Data are presented as the mean \pm SEM. All experiments were performed at least three times.

\section{Results}

RU486 decreases the survival rate of mice after trauma stress. Fourty mice were divided into two groups randomly and equally. Mice in Group 1 merely received surgery, while mice in Group 2 were treated with RU486 before surgery. After surgery, no death was observed in Group 1. However, in Group 2, the survival rate was $90 \%$ within $6 \mathrm{~h}$ after surgery and descended to $55 \%$ after $12 \mathrm{~h}$. Nearly two-thirds of the mouse in Group 2 died on Day 1, and no more death was observed beyond Day 3. The overall survival rate in Group 2 was significantly lower than that in Group 1 during 7 days of observation (20 vs. 100\%, respectively) (Fig. 1). These results indicate the protective role of endogenous glucocorticoids in the trauma mouse model.

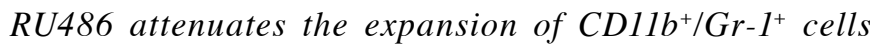
after trauma in the spleen. It has been demonstrated that an expansion of $\mathrm{CD}_{11 \mathrm{~b}} / \mathrm{Gr}-1^{+}$cells in the spleen is induced by traumatic stress (15), and the activation of the hypothalamicpituitary-adrenal axis (HPA) after trauma results in massive glucocorticoid release (22). In order to reveal the effect of endogenous glucocorticoids on TIMDSCs, CD11 $\mathrm{b}^{+} / \mathrm{Gr}-1^{+}$ splenocytes were analyzed in Group 2 (traumatic stress) and Group 3 (traumatic stress plus RU486) using flow cytometry. We found that the expansion of $\mathrm{CD} 11 \mathrm{~b}^{+} / \mathrm{Gr}-1^{+}$splenocytes was 
A

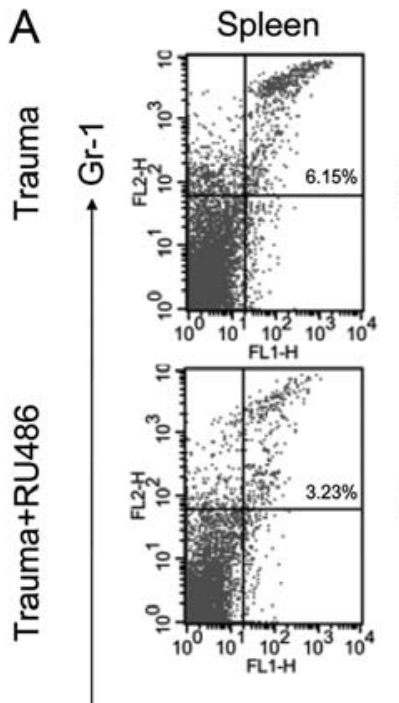

Blood
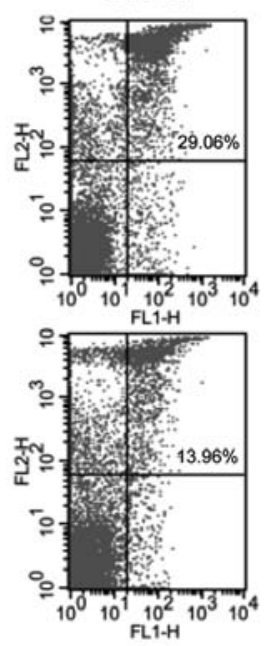

Bone marrow
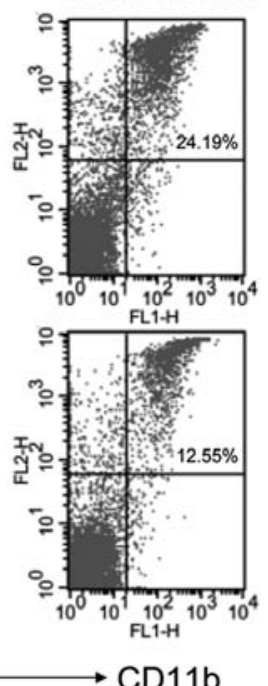

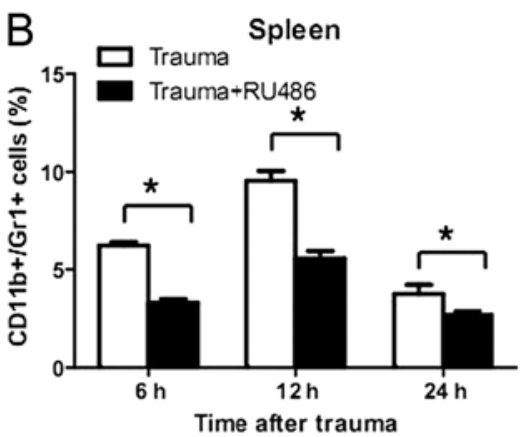

Blood

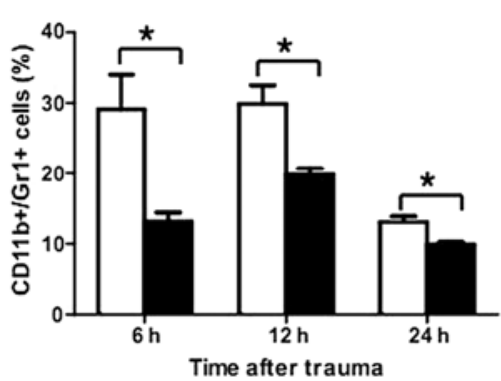

Bone marrow

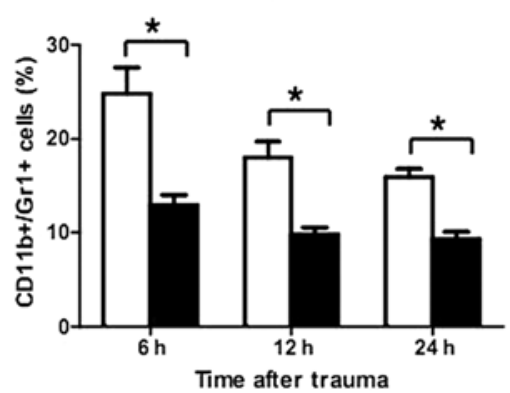

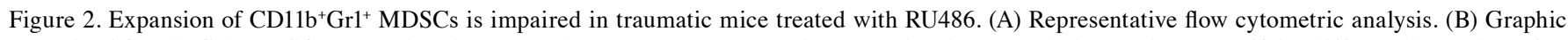
analysis of $\mathrm{CD} 11 \mathrm{~b}^{+} \mathrm{Gr} 1^{+}$MDSCs proportions in spleens, blood and bone marrow in traumatic mice treated with or without RU486 at different time points after trauma ( $\mathrm{n}=4$ per time point per goup). Date are expressed as means $\pm \mathrm{SD}$. ${ }^{*} \mathrm{P}<0.05$.

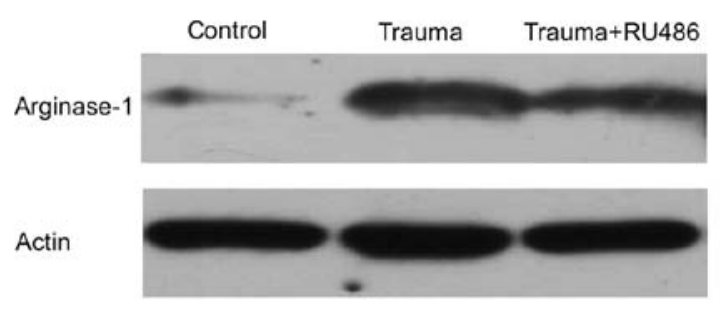

Figure 3. Arginase-1 expression in Gr-1 $1^{+}$splenocytes analyzed by western blot analysis. Liver protein lysate was used as a positive control and $\beta$-actin expression was used as a semi-quantative control. Arginase-1 expression in $\mathrm{Gr}-1^{+}$splenocytes was not obviously altered by RU486 administration in traumatic mice.

attenuated by the administration of RU486 (Fig. 2). The data suggests that massive glucocorticoid release after trauma may be involved in the expansion of $\mathrm{CD} 11 \mathrm{~b}^{+} / \mathrm{Gr}-\mathrm{l}^{+}$cells in spleen. Besides the above-mentioned experiments, immunohistochemistry was also employed to examine the $\mathrm{CD} 11 \mathrm{~b}^{+} / \mathrm{Gr}-1^{+}$cells in the spleen of mice and showed results in line with the flow cytometry data mentioned above. An apparent decrease of $\mathrm{Gr}-1^{+}$cells, which was located aroud the splenic corpuscle, was observed in the spleen of the mice treated by RU486 (Fig. 4). These results suggest that RU486 might attenuate the expansion of trauma-induced $\mathrm{CD}_{11 \mathrm{~b}} / \mathrm{Gr}-1^{+}$cells in the spleen.
RU486 inhibits the expansion of $C D 11 b^{+} / G r-1^{+}$cells after trauma in both peripheral blood and bone marrow. In our study, bone marrow cells and peripheral blood cells were also determined by flow cytometry. Bone marrow cells and peripheral blood cells were harvested and stained with anti-Gr-1 (PE) and anti-CD11b (FITC) Abs, and then were determined at 6,12 and $24 \mathrm{~h}$ after trauma stress using flow cytometry. We found that the expansion of trauma-induced $\mathrm{CD} 11 \mathrm{~b}^{+} / \mathrm{Gr}-1^{+}$cells was inhibited by RU486 in both peripheral blood and bone marrow with the most obvious inhibition at $6 \mathrm{~h}$ after trauma stress (Fig. 2). These results were consistent with the relative data observed in the spleen and indicated that endogenous glucocorticoids might be involved in TIMDSCs expansion.

RU486 does not affect the arginase-1 expression in $\mathrm{CDI}_{1 b^{+} /}$ $\mathrm{Gr}-\mathrm{I}^{+}$cells after trauma. Some reports have indicated that TIMDSCs suppressed the function of $\mathrm{T}$ cells by upregulation of arginase-1 expression, which would deplete arginine from the immune microenvironment $(15,17)$. To further determine whether RU486 can modify the characteristic of TIMDSCs, the arginase-1 expression of TIMDSCs was evaluated using western blotting. Contrary to our prediction, we found no obvious difference in arginase- 1 expression of $\mathrm{Gr}-1^{+}$splenocytes between Group 2 and Group 3 (Fig. 3). This result indicates that blockage of the glucocorticoid receptor may 
Control
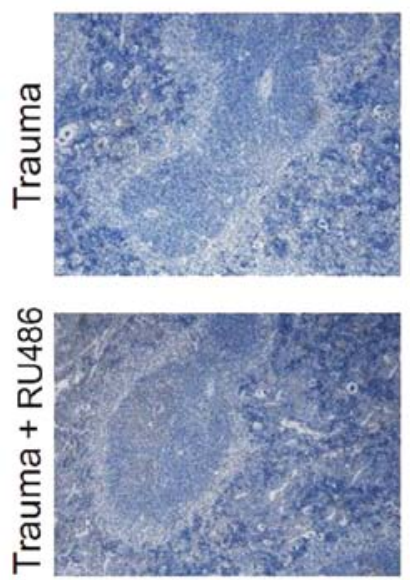

$6 \mathrm{~h}$
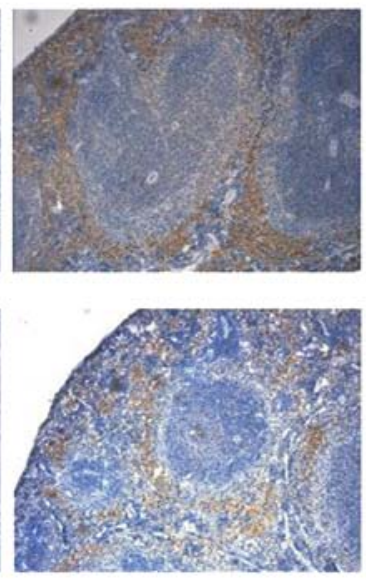

$12 \mathrm{~h}$
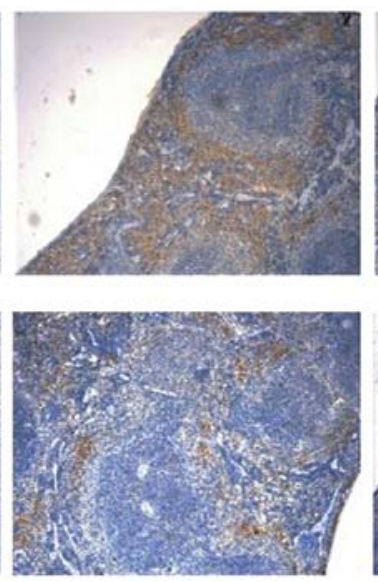

$24 \mathrm{~h}$
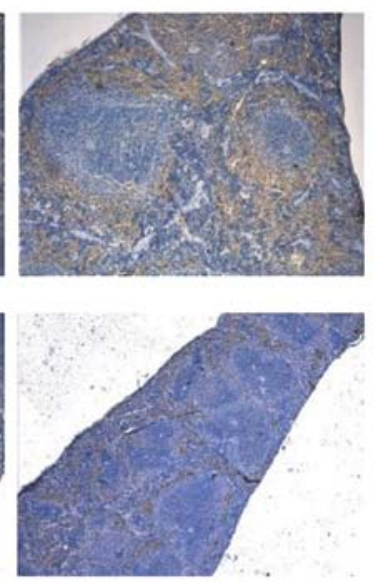

Figure 4. Accumulation of Gr- $1^{+}$cells in the spleen is blunted in traumatic mice treated with RU486. Paraffin sections were stained with Gr-1 antibody.The accumulation of Gr- $1^{+}$cells in the spleen was observed in traumatic mice within $24 \mathrm{~h}$ after trauma and was blunted by treating with RU486. Gr- $1^{+}$cells (yellow) were located at the periarteriolar lymphatic sheaths and marginal zones. There are few Gr-1 $1^{+}$cells observed in the red pulp. Magnification, x100.

Trauma Gr-1+

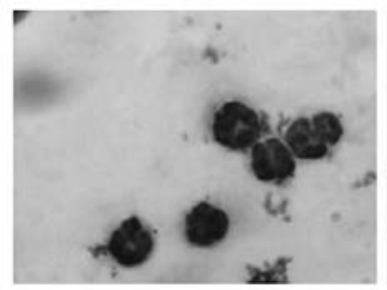

Figure 5. RU486 does not alter the morphological characterization of trauma induced MDSCs. Gr- $1^{+}$cells isolated from spleen (purity>95\%) were stained using One Step II Wright-Giemsa Stain Solution and dropped onto a slide. These cells represent a morphology resembling granulocytes or ringlike nuclei. No matter whether the traumatic mice was treated with RU486 or not, there was no difference observed in the morphological characterization of Gr- $1^{+}$splenocytes. Magnification, x400.

merely attenuate the expansion of $\mathrm{CD} 11 \mathrm{~b}^{+} / \mathrm{Gr}-1^{+}$cells induced by trauma without affecting their suppressive nature.

RU486 dose not alter the morphological characterization of trauma induced $C D 11 b^{+} / G r-1^{+}$cells. In order to further characterize the effect of RU486 on TIMDSCs, we also investigated the morphological characterization of $\mathrm{Gr}-1^{+}$splenocytes sorted by Gr-1 magnetic microbeads. Enriched Gr- $1^{+}$splenocytes were stained by One Step II Wright-Giemsa Stain Solution (Criterion Sciences). There was no obvious difference in the morphological characterization of $\mathrm{Gr}-1^{+}$splenocytes between Group 2 and Group 3, and these cells either displayed a classical morphology resembling neutrophils or showed ringed-shaped nuclei (Fig. 5), which was consistent with a previous study $(15,18)$. This result suggests that endogenous glucocorticoids may have no effect on the morphological characterization of TIMDSCs.

\section{Discussion}

Our study provided the first evidence that blockage of glucocorticoid receptors could blunt TIMDSCs expansion, which was observed in the spleen as well as in the bone marrow and peripheral blood in a murine trauma model. In addition, the arginase- 1 expression and the morphological characterization of TIMDSCs was not affected by RU486 administration. Moreover, blockage of glucocorticoid receptors decreased the survival rate of traumatic mice while it attenuated TIMDSCs expansion. These results indicate that endogenous glucocorticoids may promote TIMDSCs expansion without changing their characteristics and TIMDSCs may exert protective effects on trauma host.

It is well known that the hypothalamic-pituitary-adrenal (HPA) axis is activated after trauma, and then, massive endogenous glucocorticoid is released and plays a protective role in trauma host (22-24). The underlying mechanisms involved in this protective effect include inhibiting pro-inflammatory cytokines and promoting anti-inflammatory cytokines $(25,26)$. Moreover, glucocorticoids exert an effect on some immunoregulatory cells, such as immunoregulatory $\mathrm{T}$ cell (Treg) and MDSCs $(19,26,27)$. Previous studies have demonstrated an accumulation of MDSCs in spleen after trauma $(15,16,28,29)$. However, the effect of endogenous glucocorticoids on MDSCs in the trauma host has not been presented to date.

In this study, a murine trauma model was used to determine the effect of RU486 on TIMDSCs. We found that TIMDSCs accumulation in the spleen was attenuated by RU486 administration, and TIMDSCs were in the same location of the spleen as described by previous reports (14,15,30) (Figs. 2 and 4). Moreover, exogenous glucocorticoids have been reported to induce MDSCs proliferation in both human and mouse in vitro (19-21). Furthermore, in vivo studies have demonstrated the promotive effect of glucocorticoids on MDSCs in endotoxin immunosuppressed mice (31). In our study, being consistent with previous studies, endogenous glucocorticoids exerted similar effects on MDSCs in the spleen of a murine trauma model (Figs. 2 and 4). However, the majority of previous studies about TIMDSCs focus on the spleen, while little research has been conducted on TIMDSCs in other organs, such as in the peripheral blood and bone marrow. Therefore, we further determined the percentage of TIMDSCs in the 
bone marrow and peripheral blood. We observed similar effects of endogenous glucocorticoids on TIMDSCs in the bone marrow and peripheral blood as we found in splenocytes (Fig. 2). It is of note that endogenous glucocorticoids are not the only responsible factor for TIMDSCs expansion. Previous studies in tumor-bearing host demonstrated that some trauma related inflammatory factors including S100A9/8 (32-34) and prostaglandin E2 (PGE2) (35-37) played an important role in MDSCs expansion. Therefore, these factors may also promote TIMDSCs expansion in trauma host, besides glucocorticoids.

Under traumatic conditions, excess neutrophils as well as neutrophil-like TIMDSCs (Fig. 5) are mobilized and activated (1). However, inappropriate neutrophil sequestration in the lung often leads to hospital-acquired pneumonia and finally to pneumonia or even acute respiratory distress syndrome (ARDS) in trauma patients $(6,38,39)$. In a multicenter, randomized, double-blind, placebo-controlled study, investigators have elucidated that stress-dose of corticosteroid decreases the risk of hospital-acquired pneumonia in trauma patients with corticosteroid insufficiency (6). In our study, mimicking corticosteroid insufficiency, blockage of the glucocorticoid receptor with RU486 decreased the survival rate of traumatized mice while it inhibited TIMDSCs expansion (Figs. 1 and 2). We thus infer that TIMDSCs may limit SIRS after trauma via expressing immunosuppressive factors such as arginase-1. In addition, the expansion of neutrophil-like TIMDSCs may lead to the descent of pro-inflammatory neutrophils, since both TIMDSCs and neutrophils may be derived from the same progenitor. Taken together, it seems that TIMDSCs expansion promoted by endogenous gulcocorticoids may play a beneficial role in the trauma host.

Since TIMDSCs regulate the immune response via producing arginase-1, which depletes arginine within the immune environment and results in arginine deficiency followed by $\mathrm{T}$ cell dysfunction (15), we further determined the production of arginase-1 in TIMDSCs. Western blotting found no effect of RU486 on arginase production (Fig. 3), indicating that endogenous glucocorticoids may not alter the suppressive activity of TIMDSCs though affecting their number and percentage. This discrepancy may be due to the difference between the signal pathways for MDSCs expansion and those for activation $(11,40)$.

In summary, this study presents the first evidence that RU486 can blunt TIMDSCs expansion, which may reveal the relation between endogenous glucocorticoids and TIMDSC in vivo. The present study shows that endogenous glucocorticoids promote TIMDSCs expansion, and TIMDSCs may play a beneficial role in trauma host. Our study provides a possible mechanism underlying the protective effects of endogenous glucocorticoids in trauma patients.

\section{Acknowledgements}

This study was supported by a grant from the National Natural Science Foundation of China (No. 303069218).

\section{References}

1. Lenz A, Franklin GA and Cheadle WG: Systemic inflammation after trauma. Injury 38: 1336-1345, 2007.
2. Osborn TM, Tracy JK, Dunne JR, Pasquale M and Napolitano LM: Epidemiology of sepsis in patients with traumatic injury. Crit Care Med 32: 2234-2240, 2004.

3. Nast-Kolb D, Aufmkolk M, Rucholtz S, Obertacke U and Waydhas C: Multiple organ failure still a major cause of morbidity but not mortality in blunt multiple trauma. J Trauma 51: 835-842, 2001.

4. Hoover L, Bochicchio GV, Napolitano LM, et al: Systemic inflammatory response syndrome and nosocomial infection in trauma. J Trauma 61: 310-317, 2006.

5. Keh D, Boehnke T, Weber-Cartens S, et al: Immunologic and hemodynamic effects of 'low-dose' hydrocortisone in septic shock: a double-blind, randomized, placebo-controlled, crossover study. Am J Respir Crit Care Med 167: 512-520, 2003.

6. Roquilly A, Mahe PJ, Seguin P, et al: Hydrocortisone therapy for patients with multiple trauma: the randomized controlled HYPOLYTE study. JAMA 305: 1201-1209, 2011.

7. Hoen S, Asehnoune K, Brailly-Tabard S, et al: Cortisol response to corticotropin stimulation in trauma patients: influence of hemorrhagic shock. Anesthesiology 97: 807-813, 2002.

8. Confalonieri M, Urbino R, Potena A, et al: Hydrocortisone infusion for severe community-acquired pneumonia: a preliminary randomized study. Am J Respir Crit Care Med 171: 242-248, 2005.

9. Rhen T and Cidlowski JA: Antiinflammatory action of glucocorticoids - new mechanisms for old drugs. N Engl J Med 353: 1711-1723, 2005.

10. Ostrand-Rosenberg S and Sinha P: Myeloid-derived suppressor cells: linking inflammation and cancer. J Immunol 182: 4499-4506, 2009.

11. Gabrilovich DI and Nagaraj S: Myeloid-derived suppressor cells as regulators of the immune system. Nat Rev Immunol 9: 162-174, 2009.

12. Ochoa AC, Zea AH, Hernandez C and Rodriguez PC: Arginase, prostaglandins, and myeloid-derived suppressor cells in renal cell carcinoma. Clin Cancer Res 13: S721-S726, 2007.

13. Zhu B, Bando Y, Xiao S, et al: CD11b+Ly-6C(hi) suppressive monocytes in experimental autoimmune encephalomyelitis. J Immunol 179: 5228-5237, 2007.

14. Delano MJ, Scumpia PO, Weinstein JS, et al: MyD88-dependent expansion of an immature GR-1(+)CD11b(+) population induces $\mathrm{T}$ cell suppression and Th2 polarization in sepsis. J Exp Med 204: 1463-1474, 2007.

15. Makarenkova VP, Bansal V, Matta BM, Perez LA and Ochoa JB: $\mathrm{CD}_{11 \mathrm{~b}}{ }^{+} / \mathrm{Gr}-1^{+}$myeloid suppressor cells cause $\mathrm{T}$ cell dysfunction after traumatic stress. J Immunol 176: 2085-2094, 2006.

16. Munera V, Popovic PJ, Bryk J, et al: Stat 6-dependent induction of myeloid derived suppressor cells after physical injury regulates nitric oxide response to endotoxin. Ann Surg 251: 120-126, 2010.

17. Bryk JA, Popovic PJ, Zenati MS, Munera V, Pribis JP and Ochoa JB: Nature of myeloid cells expressing arginase 1 in peripheral blood after trauma. J Trauma 68: 843-852, 2010.

18. Cuenca AG, Delano MJ, Kelly-Scumpia KM, et al: A paradoxical role for myeloid-derived suppressor cells in sepsis and trauma. Mol Med 17: 281-292, 2011.

19. Varga G, Ehrchen J, Tsianakas A, et al: Glucocorticoids induce an activated, anti-inflammatory monocyte subset in mice that resembles myeloid-derived suppressor cells. J Leukoc Biol 84: 644-650, 2008

20. Ehrchen J, Steinmuller L, Barczyk K, et al: Glucocorticoids induce differentiation of a specifically activated, anti-inflammatory subtype of human monocytes. Blood 109: 1265-1274, 2007.

21. Barczyk K, Ehrchen J, Tenbrock K, et al: Glucocorticoids promote survival of anti-inflammatory macrophages via stimulation of adenosine receptor A3. Blood 116: 446-455, 2010.

22. Chrousos GP: The hypothalamic-pituitary-adrenal axis and immune-mediated inflammation. N Engl J Med 332: 1351-1362, 1995.

23. Kashiwabara M, Miyashita M, Nomura T, et al: Surgical traumainduced adrenal insufficiency is associated with postoperative inflammatory responses. J Nihon Med Sch 74: 274-283, 2007.

24. Marik PE and Zaloga GP: Adrenal insufficiency during septic shock. Crit Care Med 31: 141-145, 2003.

25. Chinenov Y and Rogatsky I: Glucocorticoids and the innate immune system: crosstalk with the toll-like receptor signaling network. Mol Cell Endocrinol 275: 30-42, 2007.

26. Baschant U and Tuckermann J: The role of the glucocorticoid receptor in inflammation and immunity. J Steroid Biochem Mol Biol 120: 69-75, 2010. 
27. Chen X, Murakami T, Oppenheim JJ and Howard OM: Differential response of murine $\mathrm{CD} 4^{+} \mathrm{CD} 25^{+}$and $\mathrm{CD} 4^{+} \mathrm{CD} 25^{-} \mathrm{T}$ cells to dexamethasone-induced cell death. Eur J Immunol 34: 859-869, 2004

28. Barrera G, Landoni V, Martire-Greco D, et al: Model of polymicrobial peritonitis that induces the proinflammatory and immunosuppressive phases of sepsis. Infect Immun 79: 1280-1288, 2011.

29. Schwacha MG, Thobe BM, Daniel T and Hubbard WJ: Impact of thermal injury on wound infiltration and the dermal inflammatory response. J Surg Res 158: 112-120, 2010

30. Sander LE, Sackett SD, Dierssen U, et al: Hepatic acute-phase proteins control innate immune responses during infection by promoting myeloid-derived suppressor cell function. J Exp Med 207: 1453-1464, 2010

31. Rearte B, Maglioco A, Balboa L, et al: Mifepristone (RU486) restores humoral and $\mathrm{T}$ cell-mediated immune response in endotoxin immunosuppressed mice. Clin Exp Immunol 162: 568-577, 2010

32. Ichikawa M, Williams R, Wang L, Vogl T and Srikrishna G: S100A8/A9 activate key genes and signaling pathways in colon tumor progression. Mol Cancer Res 9: 133-148, 2011.

33. Cheng P, Corzo CA, Luetteke N, et al: Inhibition of dendritic cell differentiation and accumulation of myeloid-derived suppressor cells in cancer is regulated by S100A9 protein. J Exp Med 205: 2235-2249, 2008.
34. Sinha P, Okoro C, Foell D, Freeze HH, Ostrand-Rosenberg S and Srikrishna G: Proinflammatory S100 proteins regulate the accumulation of myeloid-derived suppressor cells. J Immunol 181: 4666-4675, 2008

35. Fujita M, Kohanbash G, Fellows-Mayle W, et al: COX-2 blockade suppresses gliomagenesis by inhibiting myeloid-derived suppressor cells. Cancer Res 71: 2664-2674, 2011.

36. Eruslanov E, Daurkin I, Vieweg J, Daaka Y and Kusmartsev S: Aberrant PGE metabolism in bladder tumor microenvironment promotes immunosuppressive phenotype of tumor-infiltrating myeloid cells. Int Immunopharmacol 11: 848-855, 2011.

37. Sinha P, Clements VK, Fulton AM and Ostrand-Rosenberg S: Prostaglandin E2 promotes tumor progression by inducing myeloid-derived suppressor cells. Cancer Res 67: 4507-4513, 2007.

38. Geerts L, Jorens PG, Willems J, De Ley M and Slegers H: Natural inhibitors of neutrophil function in acute respiratory distress syndrome. Crit Care Med 29: 1920-1924, 2001.

39. Chollet-Martin S, Jourdain B, Gibert C, Elbim C, Chastre J and Gougerot-Pocidalo MA: Interactions between neutrophils and cytokines in blood and alveolar spaces during ARDS. Am J Respir Crit Care Med 154: 594-601, 1996.

40. Condamine T and Gabrilovich DI: Molecular mechanisms regulating myeloid-derived suppressor cell differentiation and function. Trends Immunol 32: 19-25, 2011. 